\title{
ORAL COMMUNICATIONS
}

OPENING CEREMONY

KEYNOTE LECTURES

Monday July 6

Tuesday July 7

Wednesday July 8

SYMPOSIA AND WORKSHOPS

Sunday July 5

Monday July 6

Tuesday July 7

Wednesday July 8

Thursday July 9

ABSTRACTS BASED ORAL COMMUNICATIONS

Monday July 6

Tuesday July 7

Wednesday July 8

\section{SATELLITE INDUSTRY SESSIONS}

Sunday July 5

Monday July 6

Tuesday July 7

Wednesday July 8

\section{POSTERS}

Monday July 6

Track A - Biological Sciences

Track B - Health Sciences and Geriatric Medicine

Track C - Behavioural and Psychological Sciences

Track D - Social Research, Policy and Practice

Tuesday July 7

Track A - Biological Sciences

Track B - Health Sciences and Geriatric Medicine

Track C - Behavioural and Psychological Sciences

Track D - Social Research, Policy and Practice

Wednesday July 8

Track A - Biological Sciences

Track B - Health Sciences and Geriatric Medicine

Track C - Behavioural and Psychological Sciences

Track D - Social Research, Policy and Practice 